\title{
ARHGAP24 ameliorates inflammatory response through inactivating Rac1/Akt/NF-кB pathway in acute pneumonia model of rat
}

\author{
Huailian Liu ${ }^{1}$, Wangpeng Wang ${ }^{2}$, Wenyi Shen ${ }^{3}$, Lili Wang ${ }^{3}$, Yangsong Zuo ${ }^{3}$ \\ ${ }^{1}$ Hospital Department, Huaian City Maternal and Child Health Hospital, Huaian, China; ${ }^{2}$ Central Laboratory, Lianshui County People's Hospital, \\ Huaian, China; ${ }^{3}$ Aspiration Medicine, Lianshui County People's Hospital, Huaian, China \\ Contributions: (I) Conception and design: H Liu; (II) Administrative support: W Wang; (III) Provision of study materials or patients: W Shen; (IV) \\ Collection and assembly of data: L Wang; (V) Data analysis and interpretation: Y Zuo; (VI) Manuscript writing: All authors; (VII) Final approval of \\ manuscript: All authors. \\ Correspondence to: Yangsong Zuo. Department of Aspiration Medicine, Lianshui County People’s Hospital, Huaian 223400, China. Email: 126zys@sina.com.
}

Background: ARHGAP24 might play a protective effect in the development of acute pneumonia, but the underlying mechanism remained a mystery. We aimed to investigate the effect of ARHGAP24 and explore the protective mechanism based on the acute pneumonia model of rats.

Methods: Western blotting analysis was conducted to measure the expression of ARHGAP24 in the rat model of bacillus pyocyaneus-induced acute pneumonia after 12, 24, 36, and $48 \mathrm{~h}$ modeling. In the acute pneumonia model of rat, lung histopathological change, lung edema, and levels of inflammatory cytokines in the broncho alveolar lavage fluid (BALF) were respectively measured to comprehensively evaluate the beneficial effect of overexpression of ARHGAP24 mediated by adenovirus. The western blotting analysis was conducted to evaluate Rac1/Akt/NF- $\kappa$ B pathway-related protein expression change with ARHGAP24 overexpression.

Results: We found that ARHGAP24 expression tended to be lower in the acute pneumonia model of the rat after bacillus pyocyaneus treated 12, 24, 36, and $48 \mathrm{~h}$. High expression of ARHGAP24 and a substantial ARHGAP24 positive area was found in the western blotting analysis and immunohistochemical staining in rats transfected with ARHGAP24. In the meantime, overexpression of ARHGAP24 suppressed the development of acute pneumonia through alleviating lung histopathological deterioration, lung edema, and levels of inflammatory cytokines in the BALF of the lung. What is more critical, ARHGAP24 overexpression inhibits the activation of Rac1, Akt, and NF- $\kappa$ B.

Conclusions: Thus, we conclude that ARHGAP24 ameliorated the inflammatory response in the acute pneumonia model of the rat through inactivating the Rac1/Akt/NF- $\mathrm{BB}$ pathway.

Keywords: ARHGAP24; pneumonia; pseudomonas aeruginosa; nuclear factor $\kappa \mathrm{B}(\mathrm{NF}-\kappa \mathrm{B})$; Rac1

Submitted May 19, 2020. Accepted for publication Oct 14, 2020.

doi: 10.21037/atm-20-5000

View this article at: http://dx.doi.org/10.21037/atm-20-5000

\section{Introduction}

Acute pneumonia is an inflammatory disease that originated from the lungs, higher in children, and older adults whose immune function is not fully developed or decreased, commonly leading to a high-level fatality (1).
Acute onset characterizes acute pneumonia, severe symptoms, and multiple complications that can easily cause circulatory system failure and seriously threaten the health of patients. Clinical symptoms include fever, cough, mental irritability, moist rales in the lung, and 


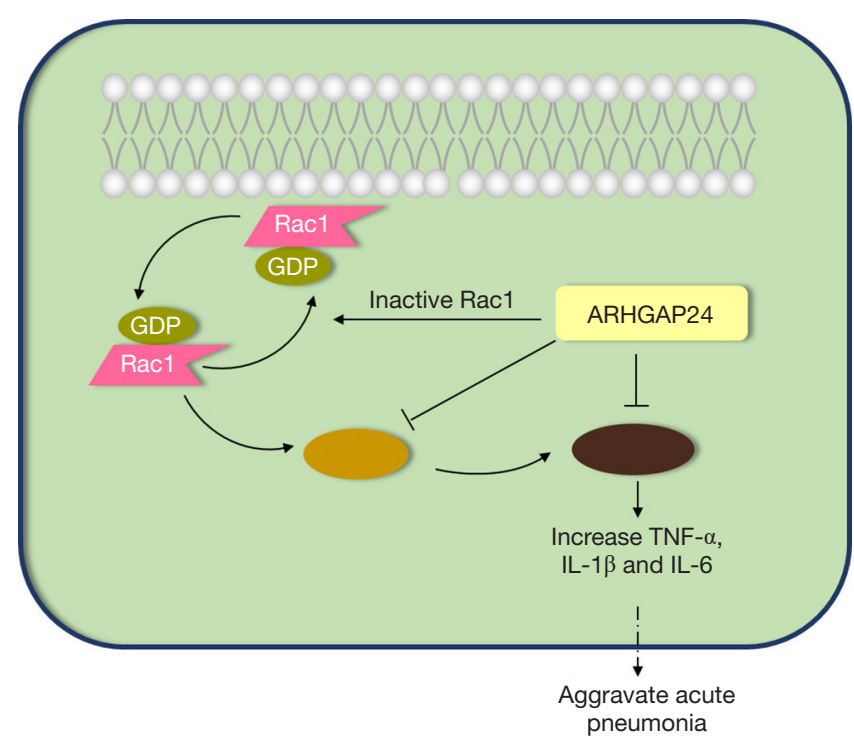

Figure 1 ARHGAP24 inactivated Rac1/Akt/NF- $\kappa$ B in acute pneumonia.

lung consolidation (2). Various microorganisms, including bacteria, viruses, chlamydia, mycoplasma, and rickettsiella, can cause acute pneumonia, the first site of which is the upper respiratory tract, bronchial infection. Severe lesions can develop to the lung segment, lung lobules, and other parts of the infection (3). As the main pathogeny of nosocomial pneumonia, pseudomona aeruginosa can cause chronic respiratory infection and even recalcitrant multidrug-resistant infections (4). Given the grim situation, new targets are urgently needed to achieve better therapy for overcoming the harmful effect of pseudomona aeruginosa induced-acute pneumonia.

Several studies have shown that activation of nuclear factor $\kappa \mathrm{B}(\mathrm{NF}-\kappa \mathrm{B})$ is a dominated event in the progression of acute pneumonia inflammation (5-7). Pro-inflammatory cytokines, including tumor necrosis factor (TNF- $\alpha$ ), can

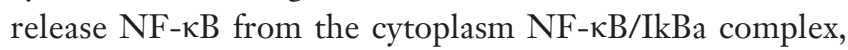
consequently causing that $\mathrm{p} 65$ quickly transfer into nuclear and react with target gene elements to launch inflammationrelated protein expression including $\mathrm{TNF}-\alpha$, interleukin $1 \beta$ (IL-1 $\beta$ ), interleukin-6 (IL-6) in the inflammatory models (8). In that case, the activation of NF- $\mathrm{KB}$ and proinflammatory cytokines will produce mutually worse the inflammation in the lung site $(9,10)$. Indeed, inhibiting the inflammation has been the target for acute pneumonia treatment for several years (11-13). Rho GTPase-activated protein 24 (ARHGAP24), a member of the GTPase family, can hydrolyze active GTP into inactive GDP and negatively regulate GTPases, including RhoA and Rac1. Previous studies have offered much evidence about the acceleration of ARHGAP24 in cell apoptosis in several cancer models (14). Notably, ARHGAP24 can play the regulatory effect through inactivating GTPase Rac1 in mouse podocytes (15). Rac1 can regulate inflammation via RAC1/ROS/NLRP3/IL-1 $\beta$ axis $(16,17)$. The inhibition of the Vav1/Rac1 pathway has been proven to facilitate the migration of macrophages (18). MARTX can inhibit intestinal inflammation via RAC1 (19). A recent study demonstrated that Rac1 activity is closely related to inflammation in lung tissue (20). What is more, Rac1/Akt/NF- $\mathrm{KB}$ has been reported to participate in the LPS-induced inflammation (21). Akt/NF-kB is the typical inflammatory signal pathway, participating in various inflammatory models (22). Inhibition of the ERK1/2 and NF- $\mathrm{BB}$ can mitigate lung inflammation in LPSinduced acute lung injury (23). Concerning the Rac1/ $\mathrm{Akt} / \mathrm{NF}-\kappa \mathrm{B}$ signal pathway and the aimed target of NF$\kappa \mathrm{B}$ in the inflammation of acute pneumonia, we assume there must be a relevant association between ARHGAP24, the upstream regulator of Rac1, and the inflammation of acute pneumonia. However, it is still mysterious about the functional role of ARHGAP24 and the potential effective mechanism involved in acute pneumonia.

The purpose of this article was to investigate the specific effect and regulatory mechanism of ARHGAP24 in Rac1/ $\mathrm{Akt} / \mathrm{NF}-\mathrm{\kappa B}$ signal pathway in the acute pneumonia model of rats (Figure 1). We have conducted lung histopathological analysis and western blotting analysis to verify our hypothesis. Our research will supply an effective target for the therapy and prognosis of acute pneumonia. We present the following article in accordance with the ARRIVE reporting checklist (available at http://dx.doi.org/10.21037/ atm-20-5000).

\section{Methods}

\section{Animals}

Male Sprague-Dawley (SD) rats, 500-650 g and 20-monthold, were obtained from Shanghai SLAC Laboratory Animal Co., Ltd. (Shanghai, China). All rats were housed in groups under clean and proper environmental conditions with enough water and food. All rats were grouped in a normal 12/12 h light/dark cycle in a room with controlled temperature $\left(22-25^{\circ} \mathrm{C}\right)$ and humidity $(50-60 \%)$. For the execution, all rats were sacrificed under $10 \%$ chloral hydrate $(300 \mathrm{mg} / \mathrm{kg}$ i.p.) and 
conducted the cervical dislocation. We have attempted to have the most minimum pain for the rats. The whole experimental protocols were following the agreement of the Laboratory Animal Committee of Hebei Medical University (Shijiazhuang, China). Experiments were performed under a project license (SCXK-20190010) granted by Hebei Medical University, in compliance with Hebei Medical institutional guidelines for the care and use of animals.

\section{Construction of expression vectors containing ARHGAP24}

The recombinant adenovirus vector for ARHGAP24 (shARHGAP24) and blank adenovirus vector was offered by Shanghai 125 Genechem. The vector was diluted to $5 \times 10^{5} / \mu \mathrm{L}$ with phosphate-buffered saline (PBS). Then, the virus with sh-ARHGAP2 4 or blank vector in $50 \mu \mathrm{L}$ was injected into the model rats of acute pneumonia via the tail vein.

\section{Experimental protocol and acute pneumonia model}

Our animal experiments were divided into two parts. First, we divided 24 rats into two groups to investigate the ARHGAP24 expression change in acute pneumonia, including a control group and model group $(n=12)$. The model group was infected with pseudomonas aeruginosa. The control group was treated with nothing. Then, an added 40 rats were divided into four groups, including the control group, model group, model + sh-ARHGAP24 group, and model + sh-con group $(\mathrm{n}=10)$. The model group, model + sh-ARHGAP24 group, and model + shcon group were infected with pseudomonas aeruginosa, except the control group. The model + sh-ARHGAP24 group and model + sh-con group were infected with pseudomonas aeruginosa promptly after adenovirusmediated transfection $6 \mathrm{~h}$. The pseudomona aeruginosa infection can be finished by injecting $0.1 \mathrm{~mL}$ bacterial suspension $\left(10^{9} \mathrm{CFU} / \mathrm{rat}\right)$ after the trachea cannula. Then, the model + sh-ARHGAP24 group was transfected with ARHGAP2 4 mediated by the adenovirus. Finally, the model + sh-con group was then transfected with a blank adenovirus vector for control.

\section{Lung wet/dry (W/D) weight ratio}

Lung wet/dry (W/D) weight ratio was measured by calculating the weight of the wet lung and dry lung. The wet lung was harvested after pseudomonas aeruginosa infection for $6 \mathrm{~h}$. The dry lung was harvested by placing it in the constant temperature drying oven at $80^{\circ} \mathrm{C}$ overnight.

\section{Broncho alveolar lavage fluid (BALF) collection}

BALF was collected through intratracheal intubation reported in the earlier study. A $5 \mathrm{~mL}$ syringe was prepared, and the tip of the needle was cut off. After anesthesia, the rats were fixed in the supine position and were exposed to the trachea. After that, a small incision was cut with scissors, and the prepared syringe needle was inserted. PBS was used for lavage, and the lavage fluidwas withdrawn with the syringe. This process can be repeated twice to obtain about $5 \mathrm{~mL}$ lavage fluid. Lavage fluid was then centrifuged at $2,000 \times \mathrm{g}$ at $4{ }^{\circ} \mathrm{C}$ for $10 \mathrm{~min}$ and stored at $-80{ }^{\circ} \mathrm{C}$ to measure inflammatory cytokines through enzyme-linked immunosorbent assay (ELISA) kits.

\section{Protein leakage}

The leaked protein in the BALF was measured by the BSA kit (Beyotime), followed by the kit protocols.

\section{ELISA analysis}

The inflammatory cytokines, including TNF- $\alpha$, IL- $1 \beta$, and IL-6, were measured by ELISA Kit (Invitrogen) according to the instruction offered by the manufacturer, all of which were measured parallelly three times.

\section{Hematein-eosin staining}

After 24 hpseudomonas aeruginosa infection, rats were euthanized for histological assessment. The lung tissue was quickly collected and placed on the ice. Then, the lung tissue was experienced with fixation by $4 \%$ paraformaldehyde for $48 \mathrm{~h}$, dehydration by gradient alcohol, and embedding by paraffin wax. After that, paraffinembedded tissue was cut into $5 \mu \mathrm{m}$ slides and placed on the glass slide, followed with dried by the oven overnight. For Hematein-Eosin staining, we firstly took off the surfaced paraffin by incubation with xylene for $20 \mathrm{~min}$. Then, we washed the slides three times with PBS and stained the slides by incubation with hematein dying (Solarbio life science) for $3 \mathrm{~min}$ and followed with eosin (Solarbio life science) for $10 \mathrm{sec}$. Finally, neutral gum closes the finished slides after air-dried and stored at room temperature. As for the pathological evaluation, slides were observed under 
a light microscope and evaluated from circumstances of edema, bleeding, and inflammation.

\section{Immunobistochemical staining}

For immunohistochemical staining, the way of obtaining lung slides has been mentioned in the "Hematein-eosin staining" part. After that, we washed the slides three times with PBS, eliminated endogenous peroxidase by incubating with $3 \%$ hydrogen peroxide for $10 \mathrm{~min}$, repaired the antigen of the tissue by incubating with tyrisin for $20 \mathrm{~min}$, and sealed the charged group of the tissue by incubating with $5 \%$ BSA for $30 \mathrm{~min}$. After these prepared work, slides were incubated with primary antibody ARHGAP24 (1:200, A-AP13826b, AmyJet Scientific) at $4{ }^{\circ} \mathrm{C}$ overnight and conjugated with the Goat Anti-Rabbit IgG H\&L $(1: 1,000, a b 6721, a b c a m)$ on the second day. Following this, lung slides were colored with a $20 \times \mathrm{DAB}$ solution $(1: 20$, Beyotime). Right After coloration, these lung slides are counterstained by hematein dying for $3 \mathrm{~min}$ and closed by neutral gum after air-dried and stored at room temperature. As for the ARHGAP24 positive fields, slides were observed under a light microscope.

\section{Immunofluorescence}

Lung tissues from the rats in acute pneumonia was collected after operation $48 \mathrm{~h}$. lung slides was harvested according to aforementioned methods. After that, we washed the slides fives times with PBS, and incubated the slides with 5\% BSA for $30 \mathrm{~min}$. After these prepared work, slides were incubated with primary antibody ARHGAP24 (1:200, A-AP13826b, AmyJet Scientific) and primary antibody SPC (1:200, ab211326, abcam) at $4{ }^{\circ} \mathrm{C}$ overnight and conjugated with the Goat Anti-Rabbit IgG-FITC (1:1,000, ab6717, abcam) on the second day. The dyeing procedure were performed in the dark. Following this, lung slides were sealed with DAPI antifade Mounting Medium (Sigma). slides were stored at 4 ${ }^{\circ} \mathrm{C}$ before observed under confocal Microscopy (TCS SP8, Leica).

\section{WB analysis}

Following the method above for collecting lung tissue, chopped lung tissue was lysed with Lysis buffer for $15 \mathrm{~min}$, and the was centrifuged at $14,000 \times \mathrm{g}$ at $4{ }^{\circ} \mathrm{C}$ for $15 \mathrm{~min}$. And then, the supernatant is collected and measured the total protein by the BSA kit. The protein was mixed with a $5 \times$ loading buffer (Beyotime) and was inactivated by boiling. Samples were detected through electrophoresis, PVDmembrane transfer, and sealing of $5 \%$ BSA. Then, samples were incubated with primary antibody ARHGAP24 (1:200, A-AP13826b, AmyJet Scientific) and at $4{ }^{\circ} \mathrm{C}$ overnight and conjugated with Goat Anti-Rabbit IgG H\&L-HRP (1:1,000, ab205718, abcam) on the second day. The expression of the protein was normalized by GADPH. Blots were detected through the ChemiDoc XRS Imaging System (Bio-Rad).

\section{Statistical analysis}

Data were expressed as mean \pm standard error of mean (SEM). We used Graphpad Prism 7 software and SPSS 22.0 software to analyze data. And we used one-way analysis of variance to conduct data statistical comparison between groups. Statistical significance was assumed at $\mathrm{P}<0.05$.

\section{Results}

\section{Down-regulation of ARHGAP24 in the lungs of acute pneumonia rats}

After Bacillus pyocyaneus treatment 12, 24, and $36 \mathrm{~h}$, we respectively collected the lung tissues and measured the ARHGAP24 expression by Wester bolt analysis (Figure 2). Western bolt analysis represented that the model group showed a significantly reduced protein expression of ARHGAP24 whenever at 12, 24, or $36 \mathrm{~h}$ when compared to the control group $(\mathrm{P}<0.05)$. Especially in $24 \mathrm{~h}$, the model group exerted a sharply lower ARHGAP24 expression compared to the control group $(\mathrm{P}<0.05)$. This phenomenon revealed that ARHGAP24 was downregulated in the lungs of acute pneumonia rats, indicating the close correlation between ARHGAP24 and acute pneumonia development.

\section{The expression of ARHGAP24 in the lungs of rats in distinct groups}

We conducted the ARHGAP24 high expression model by transfecting ARHGAP24 in the rats of acute pneumonia. In the meantime, we set the control group, acute pneumonia model group, acute pneumonia model group transfected by adenovirus vector, and acute pneumonia model group transfected by holding ARHGAP24 adenovirus. We harvested lung tissues from the four groups and measured the ARHGAP24 expression through immunohistochemical 
A

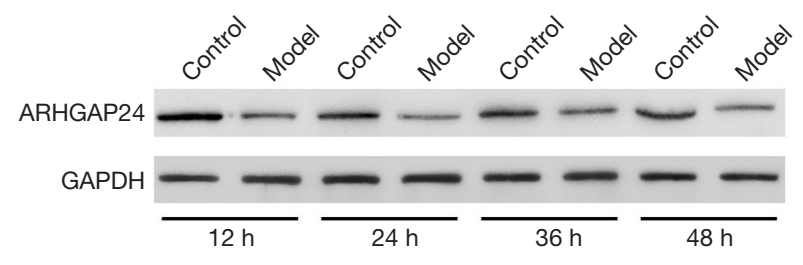

B

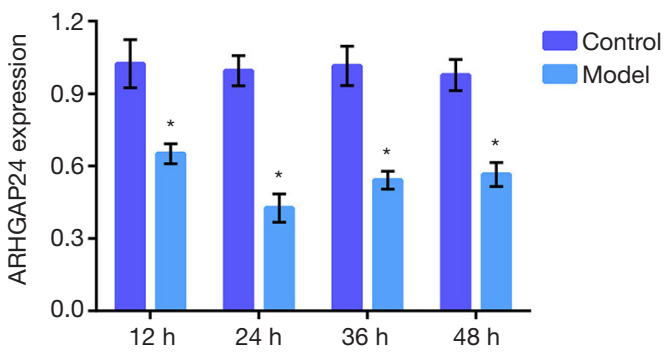

Figure 2 Down-regulation of ARHGAP24 in the lungs of acute pneumonia rats. After pseudomonas aeruginosa infection 12, 24, 36, and 48 h, the lung tissue of each group were collected to detect the expression of ARHGAP24 by WB assay. *, P<0.05 vs. control group, $\mathrm{n}=3$.

staining and $\mathrm{WB}$ analysis (Figure 3). As represented in the Figure $3 A$, the model group showed a structure of disorder and reduction of ARHGAP24 positive area compared to the control group $(\mathrm{P}<0.05)$. The same circumstances were observed in the acute pneumonia model group transfected by a blank adenovirus vector. However, the acute pneumonia model group transfected by containing ARHGAP24 adenovirus exerted a larger ARHGAP24 positive area $(\mathrm{P}<0.05)$, accompanying a standard morphological structure, which trended to be like the control group. A similar tendency was observed in the $\mathrm{WB}$ analysis (Figure 3B). The protein expression of ARHGAP24 was found to be predominantly lower in the model group than in the control group $(\mathrm{P}<0.05)$, which was following the discovery above. The protein expression of ARHGAP24 was significantly higher in the model group transfected by holding ARHGAP24 adenovirus than in the model group transfected by adenovirus vector $(\mathrm{P}<0.05)$. These statistics proved the successful building of ARHGAP24 overexpression.

\section{The effects of ARHGAP24 on the lung histopathological change and lung edema in the acute pneumonia rats}

To further investigate the effect of ARHGAP24 in acute pneumonia, we conducted a pathologic analysis by hematein eosin staining and biochemical detection (Figure 4). As pictured in Figure $4 A$, full purulent exudates were observed in the bronchi, bronchioles, and surrounding alveolar cavities in the model group, characterized as typical lobular pneumonia, the extent of which was approximately identical to the pathological situation in the model group transfected by the adenovirus vector. However, a standard and well-organized structure are observed in the model group transfected by holding ARHGAP24 adenovirus. The pathological score was significantly higher in the model group than in the control group $(\mathrm{P}<0.05)$. ARHGAP24 treatment remarkably reduced the high pathological score $(\mathrm{P}<0.05)$. Pathologic analysis showed that ARHGAP24 played a protective role in acute pneumonia.

We further verify the protective effect of ARHGAP24 by evaluating the severity degree of pulmonary edema, including measuring BALF exudate volume, protein leakage in BALF, and lung W/D weight ratio. BALF is a significant mark in the study of respiratory diseases, through which we can obtain alveolar inflammation and inflammatory cells to evaluate the degree of inflammatory lesions. As presented in Figure 4B, the model group showed a higher level of BALF exudate volume, protein leakage in BALF, and lung W/D weight ratio compared to the control group $(\mathrm{P}<0.05)$, which was accordance with pathologic analysis. Model group transfected by blank adenovirus vector still exerted the same elevated level in the BALF exudate volume, protein leakage in BALF, and lung W/D weight ratio. Importantly, ARHGAP2 4 remained to play protective effect, as evidenced by a reduced level of BALF exudate volume, protein leakage in BALF and lung W/D weight ratio were found in the model + sh-ARHGAP24 group $(\mathrm{P}<0.05)$. These statistics revealed that overexpressed ARHGAP24 mitigated pulmonary edema in rats of acute pneumonia.

\section{The effects of ARHGAP24 on the levels of inflammatory cytokines in the BALF of acute pneumonia rats}

Encouraged by a wide difference of inflammation infiltration between the model group and model group transfected by containing ARHGAP24 adenovirus in the pathological section, we hypothesized that the expression of ARHGAP24 might be involved in the inflammation reaction. Hence, we further investigate the inflammation situation by measuring the levels of TNF- $\alpha$, IL- $1 \beta$, and IL-6 in the BALF via ELISA analysis (Figure 5). TNF- $\alpha$, 

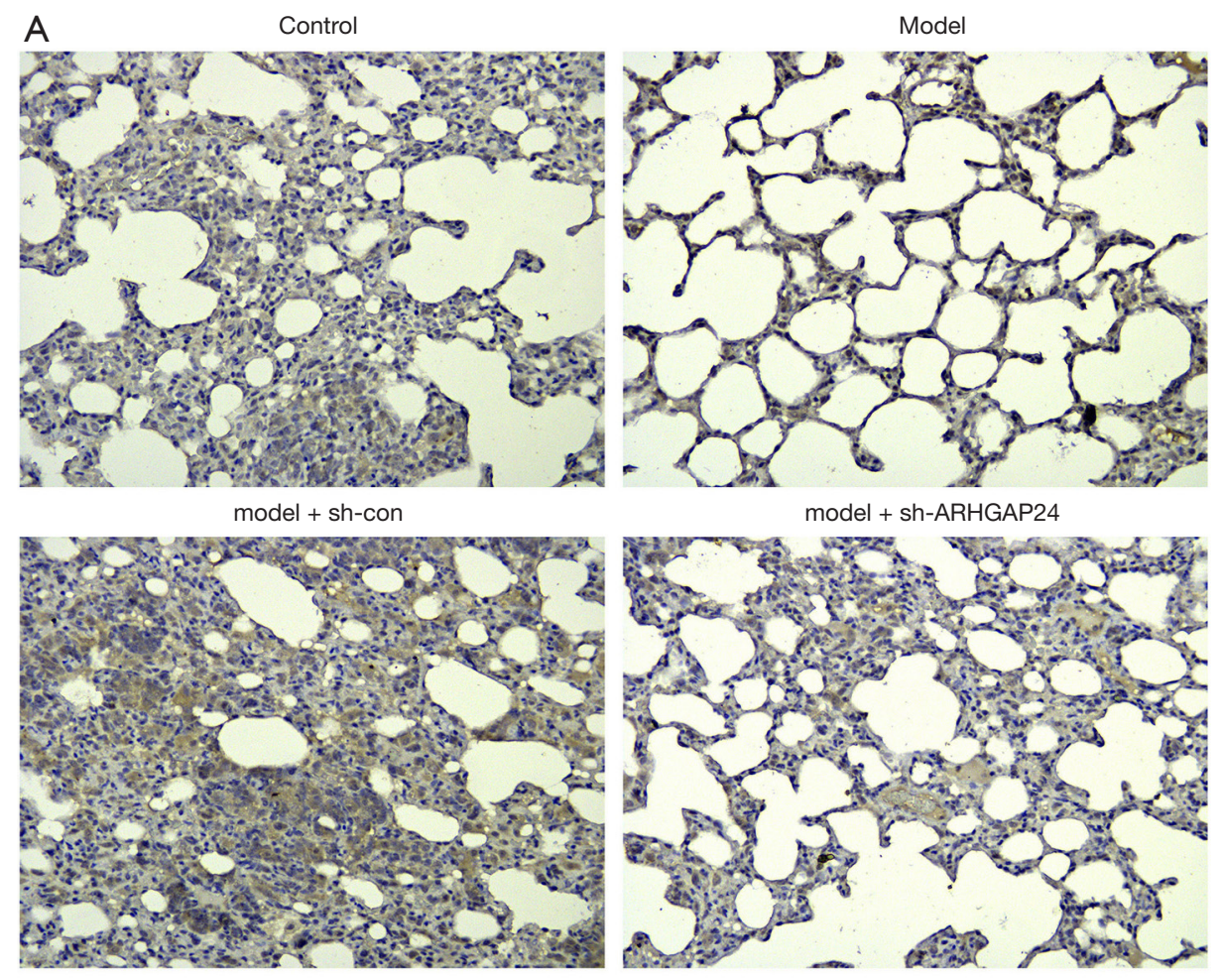

model + sh-ARHGAP24
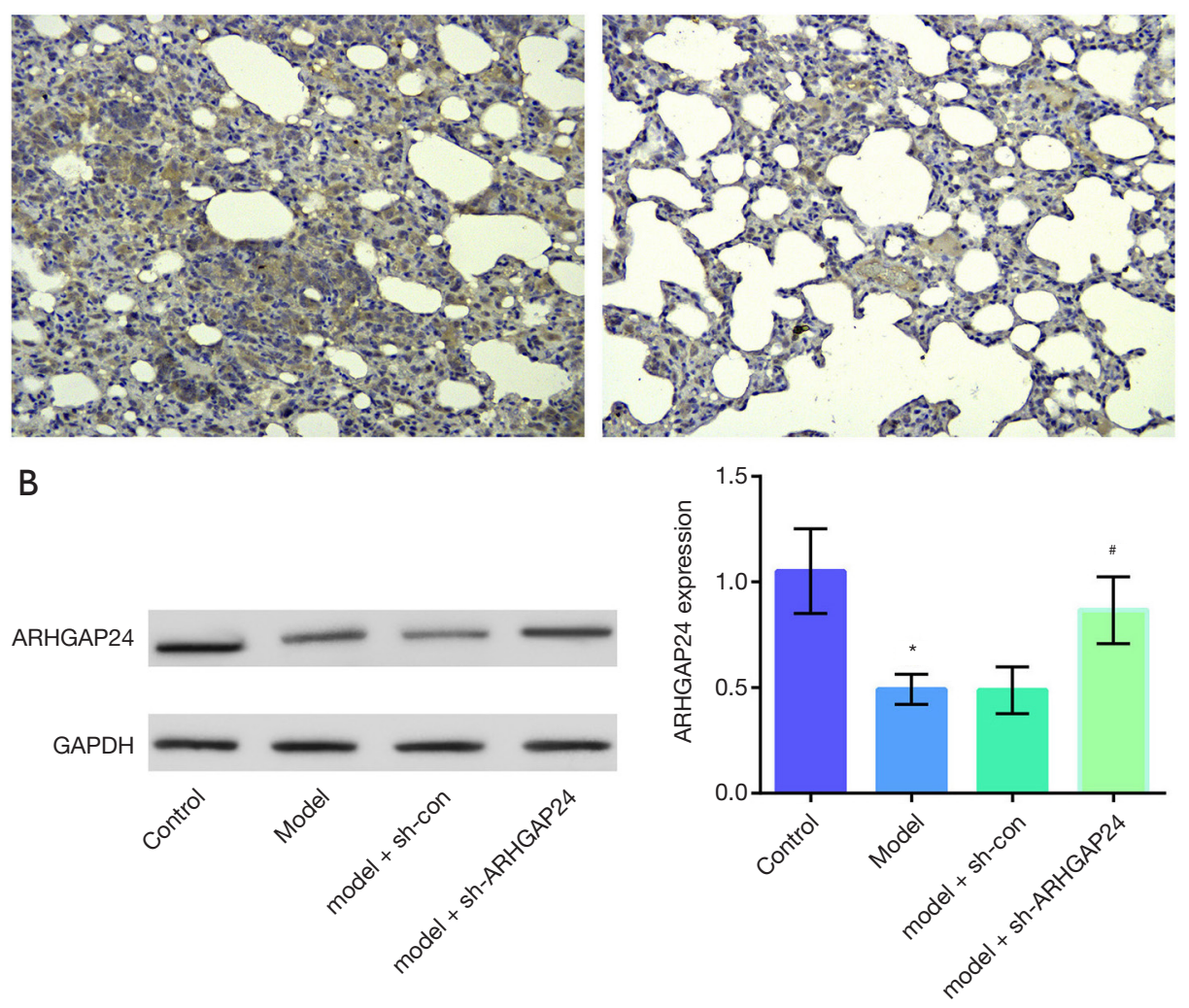

Figure 3 The expression of ARHGAP24 in the lungs of rats in different groups. (A) The immunohistochemical staining of each group was conducted to detect the expression of ARHGAP24. The scale bar represented $200 \mu \mathrm{m}$. (B) WB assay of each group was conducted to detect the expression of ARHGAP24. (C) Cartogram of the WB assay of ARHGAP24 expression. *, $\mathrm{P}<0.05$ vs. control group; * $\mathrm{P}<0.05$ vs. model + sh-con group, $\mathrm{n}=3$.

IL- $1 \beta$, and IL- 6 were typical pro-inflammatory cytokines reported to accelerate the vicious development of multiple inflammatory models, including acute pneumonia $(24,25)$. We found that the levels of TNF- $\alpha$, IL- $1 \beta$, and IL-6 were significantly higher in the model group compared to the control group $(\mathrm{P}<0.05)$, implicating the worse inflammation reaction in the lungs of acute pneumonia. Simultaneously, the similar circumstances were observed in the model group transfected by a blank adenovirus vector. Most notably, overexpression ARHGAP24 significantly decreased the levels of TNF- $\alpha$, IL- $1 \beta$, and IL-6 $(\mathrm{P}<0.05)$. These statistics showed that ARHGAP24 might alleviate the development of acute pneumonia through relieving the inflammation reaction in the lungs. 
A

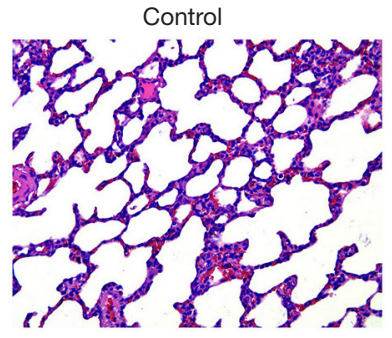

model + sh-con
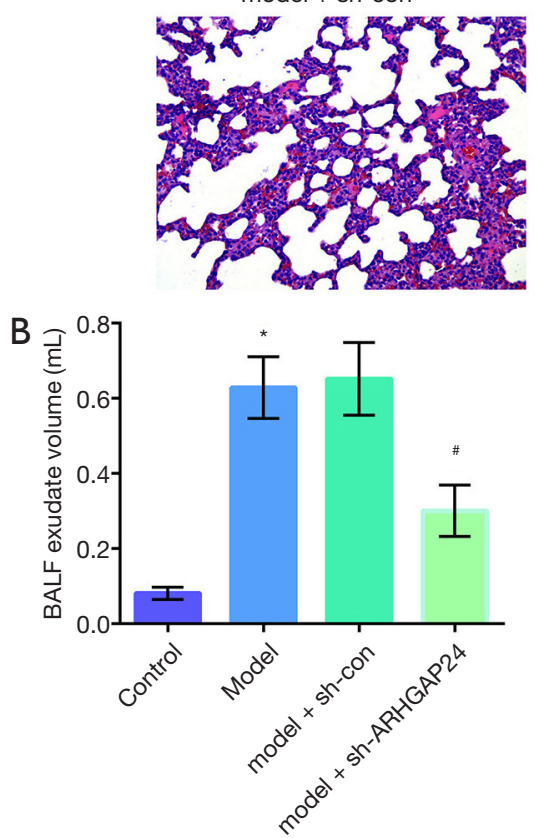

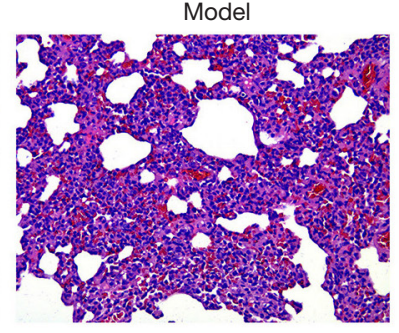

model + sh-ARHGAP24

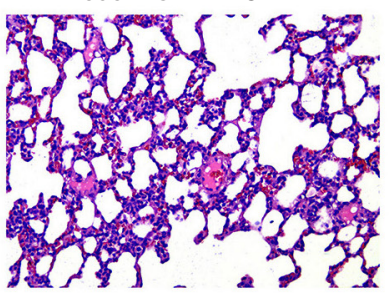

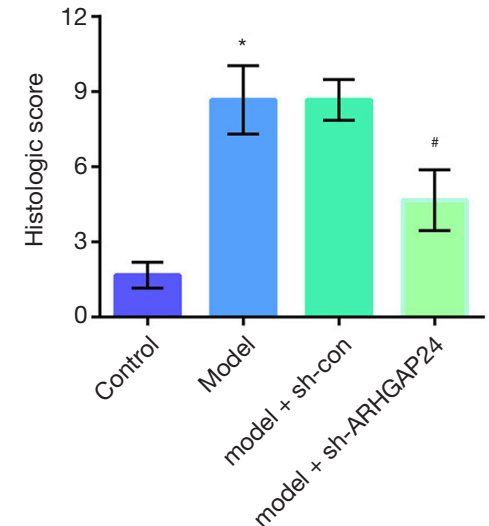
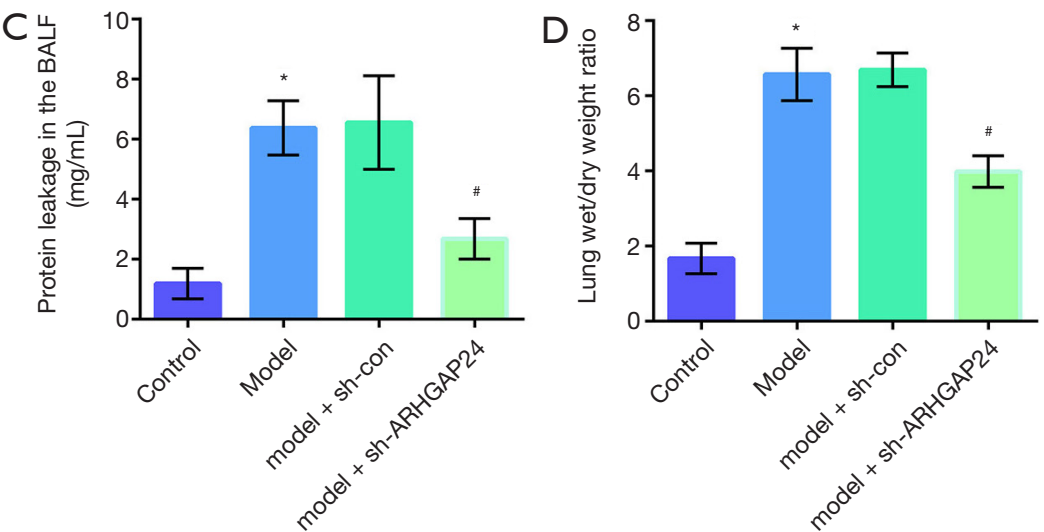

Figure 4 The effects of ARHGAP24 on the lung histopathological change and lung edema in the acute pneumonia rats. (A) The HemateinEosin staining and histological score of each group. The scale bar represented $100 \mu \mathrm{m}$. (B) The BALF level of each group. (C) The protein leakage in the BALF of each group. (D) The lung wet/dry weight of each group. *, $\mathrm{P}<0.05 v s$. control group; ${ }^{*}, \mathrm{P}<0.05 v s$. model + sh-con group, $n=3$. BALF, broncho alveolar lavage fluid.
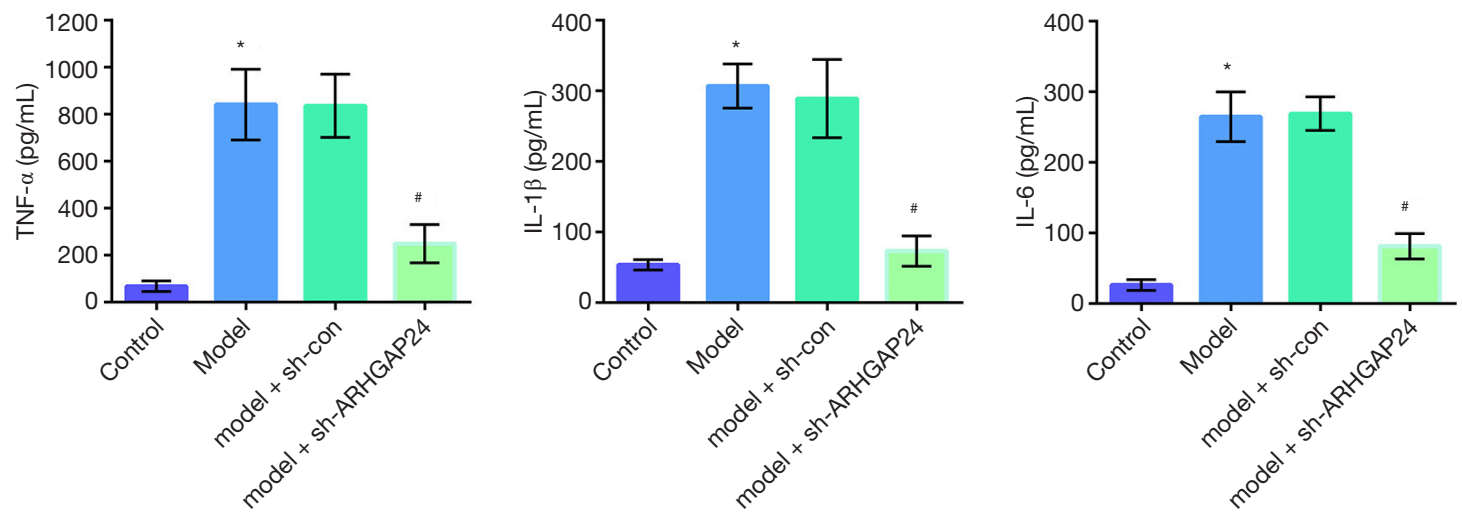

Figure 5 The effects of ARHGAP24 on the levels of inflammatory cytokines in the BALF of acute pneumonia rats. The pro-inflammatory level, including TNF- $\alpha$, IL-1 $\beta$, IL-6 of each group detected by ELISA assay. *, $\mathrm{P}<0.05$ vs. control group; ${ }^{*}, \mathrm{P}<0.05$ vs. model + sh-con group, $\mathrm{n}=3$. BALF, broncho alveolar lavage fluid; TNF- $\alpha$, tumor necrosis factor $\alpha$; IL-1 $\beta$, interleukin $1 \beta$; IL-6, interleukin-6; ELISA, ELISA, enzyme-linked immunosorbent assay. 

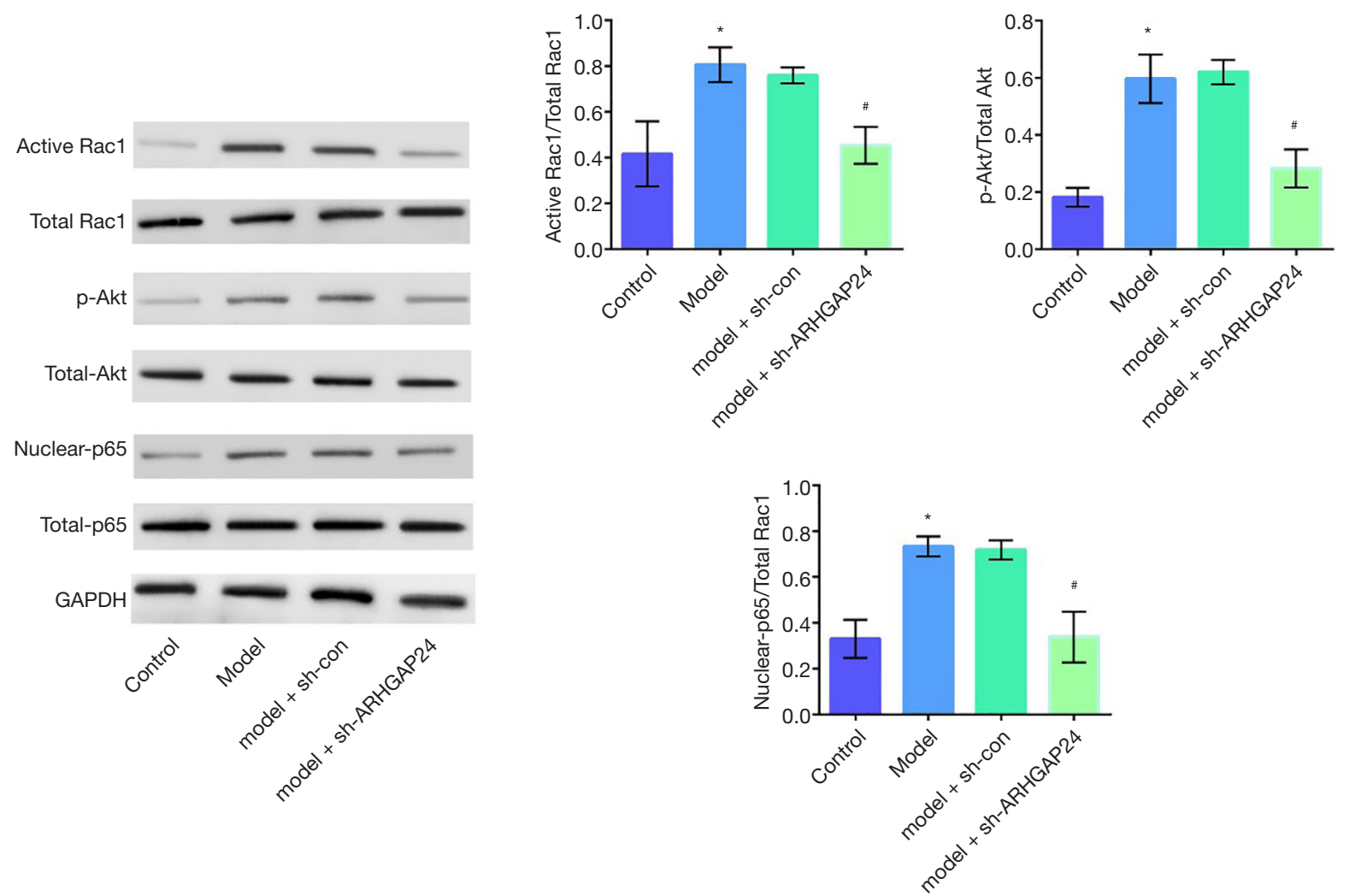

Figure 6 ARHGAP24 inactivates Rac1/Akt/NF- $\kappa$ B pathway in the lung tissues of acute pneumonia rats. The Rac1/Akt/NF- $\kappa B$ signal pathway-related protein expression via $\mathrm{WB}$ assay and statistical chart of each group in the lung. * $\mathrm{P}<0.05$ vs. control group; ${ }^{\#}, \mathrm{P}<0.05$ vs. model + sh-con group, $n=3$.

\section{ARHGAP24 inactivates the Rac1/Akt/NF-кB patbway in the lung tissues of acute pneumonia rats}

Since we have proved the protective effect of ARHGAP24 in the inflammation of lung BALF and pulmonary edema, we followed with investigating the underlying mechanism by measuring the inflammation-related protein expression by WB analysis. Therefore, the expression of Rac1, AKT, and $\mathrm{p} 65$, and their activated forms, including active Rac1, p-AKT, and nuclear p65 were respectively evaluated by WB analysis (Figure 6). The ratio of activated Rac1 and Rac1, p-AKT and AKT, nuclear-p65, and p65 were respectively represented the activated degree of Rac1, AKT, and NFKB. A higher level of ratio of activated Rac1 and Rac1, p-AKT and AKT, nuclear-p65, and p65 were observed in the model group compared to the control group $(\mathrm{P}<0.05)$, implicated the highly activated of inflammation in acute pneumonia. Simultaneously, the elevated level of ratio of activated Rac1 and Rac1, p-AKT and AKT, nuclear-p65, and p65 was observed in the model group transfected by adenovirus vector. Most surprising, a noticeable reduction was presented in the model groups transfected by containing ARHGAP2 4 adenovirus $(\mathrm{P}<0.05)$, which indicated the suppressed effect of ARHGAP24 in the Rac1, AKT, and NF-KB signal pathway. From the above statistics, we can conclude that ARHGAP24 mitigated the development of acute pneumonia through inhibiting the Rac1/Akt/NF- $\mathrm{KB}$ pathway.

\section{Discussion}

Acute pneumonia is the most contagious disease, characterized by an acute inflammatory reaction that leads to lung injury and a series of respiratory injuries $(26,27)$. Until today, oriented-inflammation therapy is still limited. We found that ARHGAP24 was downregulated in the pseudomona aeruginosa induced-acute pneumonia. Thus, we hypothesized that there might be a close relation between ARHGAP24 and the progression of acute pneumonia. Then, we successfully overexpressed the 
ARHGAP24 via adenovirus-mediated transfection in rats of acute pneumonia. Immediately after that, we conducted pathological analysis and inflammatory degree evaluation in the BALF. Therefore, the overexpression of ARHGAP24 inhibited the edema, bleeding, inflammatory evidenced by decreasing BALF exudate volume, protein leakage, and lung W/D weight ratio. This discovery might offer a promising study direction for acute pneumonia therapy.

ARHGAP24 is a Rac-specific Rho GTPase-activating protein, widely reported functional in a series of tumors (27). In clinical, a favorable overall survival accompanied the high ARHGAP24 expression in patients with astrocytoma (28). The overexpression of ARHGAP24 could result in an enhancement in cell apoptosis, followed by a high expression of p53 and bax (29). Also, the overexpression of ARHGAP24 inhibited cell invasion and elevated cell apoptosis in renal cancer (30). Importantly, a recent study reported that overexpression of ARHGAP24 remarkably inhibited cell proliferation and elevated cell apoptosis via suppressing the activity of RhoA and Rac1 in lung cancer (31). What is more, the expression of ARHGAP24 might participate in the Lung endothelial barrier disruption in Lyl1-deficient mice (32). There are few research about the effect of ARHGAP24 in the acute lung injury-induced by pseudomonas aeruginosa. Our report firstly demonstrated that overexpression of ARHGAP24 mitigated the inflammation by decreasing levels of inflammatory cytokines, including TNF- $\alpha$, IL-1 $\beta$, and IL-6 in the BALF in the rats of acute pneumonia. Thus, the antiinflammatory effect of ARHGAP24, to no insignificant extent, was achieved through Rac1.

Rac1 is the critical regulator in the inflammation signal network. Akt is a type of protein kinase B, also known as $\mathrm{PKB}$ or Rac, playing a crucial role in cell survival and apoptosis. The previous study has shown the vital effect of Rac1 and Akt in regulating cell invasion, migration, and differentiation in cancer development. It was worth noting the engagement of Rac1/Akt in the inflammation relevant signal way regulation. Inhibition of STAT1 and STAT3 exerted the anti-inflammatory effect by Rac1 inactivation (33). Activation of Rac GTPase resulted in an elevated level of anti-inflammatory cytokine IL-10 and a reduced level of pro-inflammatory, including IL-6 and TNF- $\alpha$. Specific inactivation of RhoA/Cdc42/Rac1 deceased the NF- $\mathrm{\kappa B}$ p 65 phosphorylation, the transcription, and translation of the IL-6/TNF- $\alpha$ gene in LPS-induced sepsis (34).
Moreover, the overexpression of miR-126 decreases the serum level of IL- 6 and TNF- $\alpha$ via regulating Rac1/Akt in sepsis (35). IL-6 and TNF- $\alpha$ were regulated by the NF- $\kappa B$ and could recruitment macrophages to the inflammatory sites and contributed to the secretion of cell cohesion molecules that led to the infiltration of neutrophils. Many

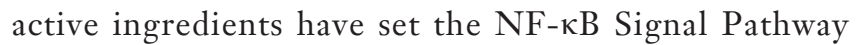
as a target to reduce inflammatory symptoms in acute lung injury (36,37). The activation of Rac1/Akt and NF$\kappa \mathrm{B}$ might be the prime culprit in the inflammation in acute pneumonia. In our article, we observed that the inactivation of Rac1/Akt $/ \mathrm{NF}-\kappa \mathrm{B}$, and consequently reduced the inflammatory cytokine level after overexpression of ARHGAP24. Thus, we can conclude that ARHGAP24 ameliorates inflammatory response through inactivating the Rac1/Akt/NF- $\mathrm{B}$ pathway in the acute pneumonia model of the rat. This relevant signal pathway in the overexpression of ARHGAP24 might be more than that. Thus, there still needs most of the research to explore the underlying mechanism.

Our research only dedicated to in vivo research, but not on in vitro research. Alveolar epithelium was an important structure involved in the mechanism of acute lung injury and rehabilitation. Increased alveolar epithelial cell (AEC) permeability was a pathological feature of lung injury, resulting in pulmonary edema, increased permeability of the epithelial barrier, and increased airway inflammation. Tissue immunolocalization by IHC though co-staining the SPC, the biomarker of AEC, and ARHGAP24 revealed that the expression distribution of ARHGAP24 was highly coincident with the expression distribution of SPC (Figure 7). This data verified our hypothesis that ARHGAP24 majorly expressed in the AEC. We suggested that the anti-inflammatory effect of ARHGAP24 in the progression of acute pneumonia in the lung tissue might be equally applicable to AEC. In order to verify the mechanism, we should investigate the anti-inflammatory ability of ARHGAP24 in the AEC in the further.

In summary, ARHGAP24 was showed to take part in regulating inflammation in rats of acute pneumonia induced by pseudomonas aeruginosa. ARHGAP24 can ameliorate inflammatory response, shown by decreasing the level of IL-6 and TNF- $\alpha$ through inactivating Rac1/Akt/NF- $\kappa$ B pathway. Overexpression of ARHGAP24 also decreased the pathological grades, BALF volume, and protein leakage. Therefore, our study supplied a new target for acute pneumonia therapy and broadened the ARHGAP24 

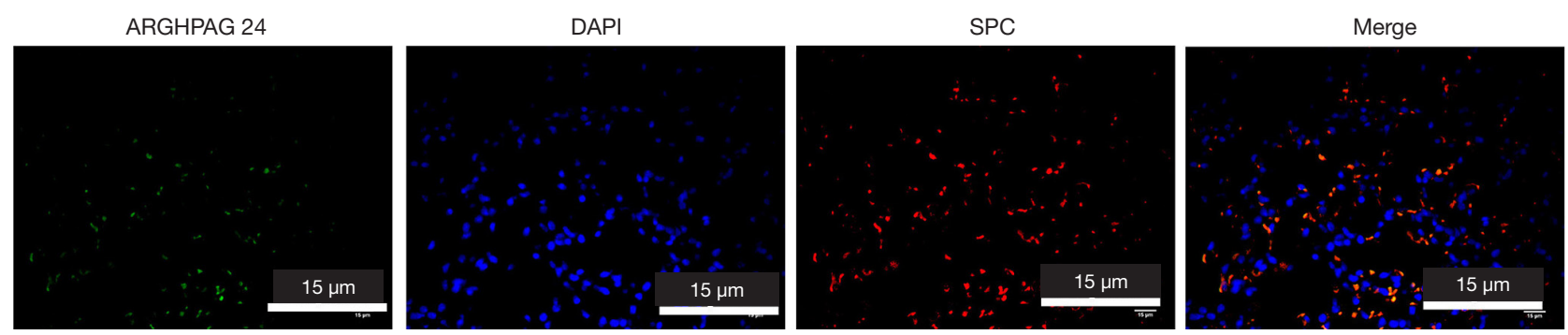

Figure 7 the expression of ARHGAP24 and SPC, the biomarkers of lung alveolar epithelial cell (AEC), were detected by tissue immunofluorescence. Green labelled the ARHGAP24, red labelled the SPC. Orange, the superposition of green and red, labelled the overlapping area of ARHGAP24 and SPC. The scale bar represented $15 \mu \mathrm{m}$.

research field.

\section{Acknowledgments}

Funding: This study was supported by the National Natural Science Foundation of China.

\section{Footnote}

Reporting Checklist: The authors have completed the ARRIVE reporting checklist. Available at http://dx.doi. org/10.21037/atm-20-5000

Data Sharing Statement: Available at http://dx.doi. org/10.21037/atm-20-5000

Conflicts of Interest: All authors have completed the ICMJE uniform disclosure form (available at http://dx.doi. org/10.21037/atm-20-5000). The authors have no conflicts of interest to declare.

Ethical Statement: The authors are accountable for all aspects of the work in ensuring that questions related to the accuracy or integrity of any part of the work are appropriately investigated and resolved. The whole experimental protocols were following the agreement of the Laboratory Animal Committee of Hebei Medical University (Shijiazhuang, China).

Open Access Statement: This is an Open Access article distributed in accordance with the Creative Commons Attribution-NonCommercial-NoDerivs 4.0 International License (CC BY-NC-ND 4.0), which permits the noncommercial replication and distribution of the article with the strict proviso that no changes or edits are made and the original work is properly cited (including links to both the formal publication through the relevant DOI and the license). See: https://creativecommons.org/licenses/by-nc-nd/4.0/.

\section{References}

1. Skřičková J. Pneumonia in immunocompromised persons. Vnitr Lek 2018;63:786-95.

2. Saldias Peñafiel F, Gassmann Poniachik J, Canelo López $\mathrm{A}$, et al. Features of community-acquired pneumonia in immunocompetent hospitalized adults according to the causal agent. Rev Med Chil 2018;146:1371-83.

3. Kolek V. Community pneumonia - fundamentals of diagnosing and treatment. Vnitr Lek 2017;63:514-7.

4. Liu C, Qi J, Shan B, et al. Pretreatment with cathelicidin$\mathrm{BF}$ ameliorates Pseudomonas aeruginosa pneumonia in mice by enhancing NETosis and the autophagy of recruited neutrophils and macrophages. Int Immunopharmacol 2018;65:382-91.

5. Feng G, Jiang ZY, Sun B, et al. Fisetin Alleviates Lipopolysaccharide-Induced Acute Lung Injury via TLR4Mediated NF- $\kappa B$ Signaling Pathway in Rats. Inflammation 2016;39:148-57.

6. Li HT, Ye C, Zhou M, et al. Moxifloxacin suppresses airway inflammation and modulates expression of caveolin-1 and flotillin-1 in airway smooth muscle cells of asthmatic rats. Ann Transl Med 2019;7:469.

7. Wu G, Zhu Q, Zeng J, et al. Extracellular mitochondrial DNA promote NLRP3 inflammasome activation and induce acute lung injury through TLR9 and NF-кB. J Thorac Dis 2019;11:4816-28.

8. Jakkampudi A, Jangala R, Reddy BR, et al. NF-kB in acute pancreatitis: Mechanisms and therapeutic potential. Pancreatology 2016;16:477-88.

9. Katsura H, Kobayashi Y, Tata PR, et al. IL-1 and TNF $\alpha$ 
Contribute to the Inflammatory Niche to Enhance Alveolar Regeneration. Stem Cell Reports 2019;12:657-66.

10. He M, Ichinose T, Yoshida S, et al. PM2.5-induced lung inflammation in mice: Differences of inflammatory response in macrophages and type II alveolar cells. J Appl Toxicol 2017;37:1203-18.

11. Zhang Y, Zhou S, Zhou J, et al. Regulation of NF-кB/ MAPK signaling pathway attenuates the acute lung inflammation in Klebsiella pneumonia rats by mollugin treatment. Microb Pathog 2019;132:369-73.

12. Dai J, Gu L, Su Y, et al. Inhibition of curcumin on influenza A virus infection and influenzal pneumonia via oxidative stress, TLR2/4, p38/JNK MAPK and NF-кB pathways. Int Immunopharmacol 2018;54:177-87.

13. Sham HP, Walker KH, Abdulnour RE, et al. 15-epiLipoxin A(4), Resolvin D2, and Resolvin D3 Induce NF- $\kappa$ B Regulators in Bacterial Pneumonia. J Immunol 2018;200:2757-66.

14. Wang L, Shen S, Wang M, et al. Rho GTPase Activating Protein 24 (ARHGAP24) Silencing Promotes Lung Cancer Cell Migration and Invasion by Activating $\beta$-Catenin Signaling. Med Sci Monit 2019;25:21-31.

15. Akilesh S, Suleiman H, Yu H, et al. Arhgap24 inactivates Rac1 in mouse podocytes, and a mutant form is associated with familial focal segmental glomerulosclerosis. J Clin Invest 2011;121:4127-37.

16. Quiros M, Feier D, Birkl D, et al. Resolvin E1 is a prorepair molecule that promotes intestinal epithelial wound healing. Proc Natl Acad Sci U S A 2020;117:9477-82.

17. Hamarsheh S, Osswald L, Saller BS, et al. Oncogenic Kras(G12D) causes myeloproliferation via NLRP3 inflammasome activation. Nat Commun 2020;11:1659.

18. Bao Z, Zhang L, Li L, et al. Ne-Carboxymethyl-Lysine Negatively Regulates Foam Cell Migration via the Vav1/ Rac1 Pathway. J Immunol Res 2020;2020:1906204.

19. Woida PJ, Satchell KJF. The Vibrio cholerae MARTX toxin silences the inflammatory response to cytoskeletal damage before inducing actin cytoskeleton collapse. Sci Signal 2020;13.

20. Li Y, Wittchen ES, Monaghan-Benson E, et al. The role of endothelial MERTK during the inflammatory response in lungs. PLoS One 2019;14:e0225051.

21. Liu J, Guo S, Jiang K, et al. miR-488 mediates negative

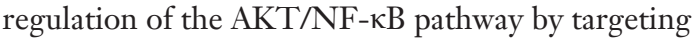
Rac1 in LPS-induced inflammation. J Cell Physiol 2020;235:4766-77.

22. Meng X, Tancharoen S, Kawahara KI, et al. 1,5-Anhydroglucitol attenuates cytokine release and protects mice with type 2 diabetes from inflammatory reactions. Int J Immunopathol Pharmacol 2010;23:105-19.

23. Li TJ, Zhao LL, Qiu J, et al. Interleukin-17 antagonist attenuates lung inflammation through inhibition of the ERK1/2 and NF- $\kappa$ B pathway in LPS-induced acute lung injury. Mol Med Rep 2017;16:2225-32.

24. Schauer AE, Klassert TE, von Lachner C, et al. IL37 Causes Excessive Inflammation and Tissue Damage in Murine Pneumococcal Pneumonia. J Innate Immun 2017;9:403-18.

25. Lin Y, Tan D, Kan Q, et al. The Protective Effect of Naringenin on Airway Remodeling after Mycoplasma Pneumoniae Infection by Inhibiting Autophagy-Mediated Lung Inflammation and Fibrosis. Mediators Inflamm 2018;2018:8753894.

26. Nicolaou EV, Bartlett AH. Necrotizing Pneumonia. Pediatr Ann 2017;46:e65-8.

27. Dietert K, Gutbier B, Wienhold SM, et al. Spectrum of pathogen- and model-specific histopathologies in mouse models of acute pneumonia. PLoS One 2017;12:e0188251.

28. Hara A, Hashimura M, Tsutsumi K, et al. The role of FilGAP, a Rac-specific Rho-GTPase-activating protein, in tumor progression and behavior of astrocytomas. Cancer Med 2016;5:3412-25.

29. Zhang S, Sui L, Zhuang J, et al. ARHGAP24 regulates cell ability and apoptosis of colorectal cancer cells via the regulation of P53. Oncol Lett 2018;16:3517-24.

30. Xu G, Lu X, Huang T, et al. ARHGAP24 inhibits cell cycle progression, induces apoptosis and suppresses invasion in renal cell carcinoma. Oncotarget 2016;7:51829-39.

31. Wang L, Shen S, Xiao H, et al. ARHGAP24 inhibits cell proliferation and cell cycle progression and induces apoptosis of lung cancer via a STAT6-WWP2-P27 axis. Carcinogenesis 2019; bgz144.

32. Pirot N, Delpech H, Deleuze V, et al. Lung endothelial barrier disruption in Lyl1-deficient mice. Am J Physiol Lung Cell Mol Physiol 2014;306:L775-85.

33. Kim HS, Cho IH, Kim JE, et al. Ethyl pyruvate has an anti-inflammatory effect by inhibiting ROS-dependent STAT signaling in activated microglia. Free Radic Biol Med 2008;45:950-63.

34. Guo F, Xing Y, Zhou Z, et al. Guanine-nucleotide exchange factor $\mathrm{H} 1$ mediates lipopolysaccharide-induced interleukin 6 and tumor necrosis factor $\alpha$ expression in endothelial cells via activation of nuclear factor $\kappa \mathrm{B}$. Shock 2012;37:531-8.

35. Wang HF, Wang YQ, Dou L, et al. Influences of up- 
regulation of miR-126 on septic inflammation and prognosis through AKT/Rac1 signaling pathway. Eur Rev Med Pharmacol Sci 2019;23:2132-8.

36. Li T, Wu YN, Wang H, et al. Dapk1 improves inflammation, oxidative stress and autophagy in LPSinduced acute lung injury via $\mathrm{p} 38 \mathrm{MAPK} / \mathrm{NF}-\kappa \mathrm{B}$ signaling pathway. Mol Immunol 2020;120:13-22.

37. Jin Y, Qian J, Ju X, et al. Osthole Protects against Acute Lung Injury by Suppressing NF-кB-Dependent Inflammation. Mediators Inflamm 2018;2018:4934592.

(English Language Editor: J. Chapnick)

Cite this article as: Liu H, Wang W, Shen W, Wang L, Zuo Y. ARHGAP24 ameliorates inflammatory response through

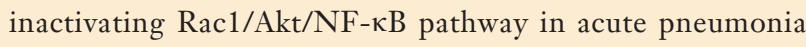
model of rat. Ann Transl Med 2020;8(20):1289. doi: 10.21037/atm20-5000 